\title{
A new method of ballistic mission target recognition
}

\author{
Li Changxi ${ }^{1, a^{*}}$, Zhou Yan ${ }^{1, b}$ and Lin $\operatorname{Han}^{2, \mathrm{c}}$ \\ ${ }^{1}$ Air Force Early Warning Academy. Wuhan, China \\ ${ }^{2}$ Dongfang College, Fujian Agriculture and Forestry University Fuzhou, China \\ alichangxi528@163.com, ${ }^{\mathrm{b}}$ 1292796462@qq.com, ${ }^{\mathrm{c}}$ 5918409@qq.com
}

Keywords:target recognition; ballistic mission; D-S theory

\begin{abstract}
On the process of target ballistic missile (BM) recognition, in other to solve the problem of multi-sensor system is easy to conflict when transmitting date, and the problem that the conflicting evidence and unconflicting evidence integration together on existing D-S improved algorithm, this paper put forward the target recognition method of ballistic mission based on experts trust, gives the model of target BM recognition system based on experts trust and the model of expert knowledge base. The new algorithm divides the collected evidence into two groups, which one is conflicting evidence set and the other is unconflicting evidence set, conflicting evidence set evaluation by expert evaluation system and unconflicting evidence set fusing by traditional D-S theory, and then refusing the two results, get the decision. By comparing with the old algorithms, experiment results show that the new algorithm is more effectively.
\end{abstract}

\section{Introduction}

With the increasing the diversity of the ballistic missile target and the complexity of modern battlefield environment, senors brought fuzziness and uncertainty of information fusion Currently, Dempster-Shafer theory of evidence (D-S theory) is one of the effective means to solve this kind of vagueness problem, but when dealing with a conflict of evidence, often obtained contrary to common sense conclusions. At present, many scholars have proposed various theories on the D-S improved methods, and its thoughts focused on the following two aspects: one is that Dempster combination rule needs to be modified to work, the literature [1-9] have done a lot, and have achieved good results; another model that the evidence you need to modify, the literature [10-12] have done a lot of work and achieved some success. In recent years, it was also put forward some compromise principles, such as the literature [13] by combining the advantages of the rules and evidence synthesis model proposed a new method to resolve conflicts of evidence fusion, also achieved good results. Although there were many correction method of the D-S, but analysed these improved algorithm carefully, we found that: on the one hand, the integration and inconsistent evidence by the weight of the problem remains the focus, on the other hand, to solve the traditional problem has brought new problems at the same time. Meanwhile, the improved algorithm still be unified the conflict evidence integration.

As we all know, for two high conflicting evidence, either one is right and one is wrong, or both are wrong, its weight distribution after integration, the error rate is still presence after integration regardless of the error distribution more than the weight of evidence is low. In multi-sensor information fusion system aimed ballistic missile target recognition, it easy to happen the situation of transmission of data from multiple sensors conflict due to disguise or interfere with measures taken by the enemy, if unified integration right now, the error rate will accumulate, and even influence the final decision. To address this issue, this paper presents a ballistic missile target recognition method based on expert trust, the method will pass over a certain time sensor information are grouped according to whether the conflict, expert identification system was used to evaluate basic probability assignment of evidence of high conflict and D-S 
theory was directly used to mix low conflict of evidence, then used the results of expert evaluation of the results of D-S theory and then fused D-S integration, obtained the target of basic probability assignment and made decisions, finally, through experiments verified the effectiveness of the algorithm.

\section{D-S theory and conflict analysis}

\section{D-S theory}

D-S theory is a mathematical theory established by Dempster Shafer and his students in the late 1960s and early 1970s. The theory first defines a framework for identification $U$, on this basis, the establishment of a mass function or basic probability assignment (BPA), which satisfies the following conditions:

$$
m(\phi)=0, \sum_{A \in U} m(A)=1
$$

where, $\phi$ is empty set. BPA means the trust of proposition A.

Suppose $m_{1}, m_{2}$ are two BPA of framework for identification $U, A_{1}, A_{2}, \cdots, A_{k}$ and $B_{1}, B_{2}, \cdots, B r$ are focal elements to be identified, so the D-S combination rule as follows:

$$
m(\phi)=0, m(C)=\frac{1}{1-\mathrm{k}_{i \in k, j \in r}} \sum_{\substack{i \\ A_{i} \cap B_{j}}} m_{1}\left(A_{k}\right) m_{2}\left(B_{r}\right) \quad C \in U, C \neq \phi
$$

where, $k=\sum_{\substack{i \in k, j, r \\ A_{i} \cap B_{j}=\phi}} m_{1}\left(A_{i}\right) m\left(B_{j}\right)<1$ means conflicting coefficient, the larger the $k$ is, the greater the conflict between evidences, and when $k=1$, combination rule was unavailable.

\section{Conflict Analysis}

Recently, according to the degree of conflict and the conflict between the characteristics of the evidence can be divided into three types of conflict: whole conflict paradox, the paradox 0 trusts, and a trust paradox.

(1) Total conflict paradox. Suppose any two identification evidence given under the framework of BPA was totally conflict, even if most of the other evidence BPA good consistency, D-S combination rule is unavailable, which was total conflict paradox. Expression (2) showed that if the conflicting coefficient $k=1$, the evidence combination rule which due to conflicting was unavailable.

(2) 0 trust paradox. Suppose a number of evidence under the framework of a particular piece of evidence to identify a focal element of BPA was 0 , and the focal element with the same evidence in the other BPA was not the focal element of the intersection was not itself, other evidence, regardless of the focal element of how much BPA, this combination results in the focal element of BPA is always 0 , which was the 0 trust paradox.

(3) 1 trust paradox. Suppose $U$ is a framework for identification $m_{i}\left(E_{j}\right)$ means the BPA of article $i$ evidence, $E j$ means focal element, $i=1,2, \cdots 1, j=1,2, \cdots \mathrm{N}$, where, 1 is the number of evidence of $\mathrm{U}, \mathrm{N} \leqslant 2 \mathrm{n}-1$ is the number of focal element, if $m_{l}\left(E_{j l}\right)=m_{2}\left(E_{j 2}\right)=\cdots=m_{l}\left(E_{j l}\right)=a \neq 0, \frown E_{j i}=\mathrm{A}$ and $k=1-a^{l}$, when a is small, the BPA of all evidence is also small, but the results of combination is 1 , which is unreasonable, this situation called 1 trust paradox. 


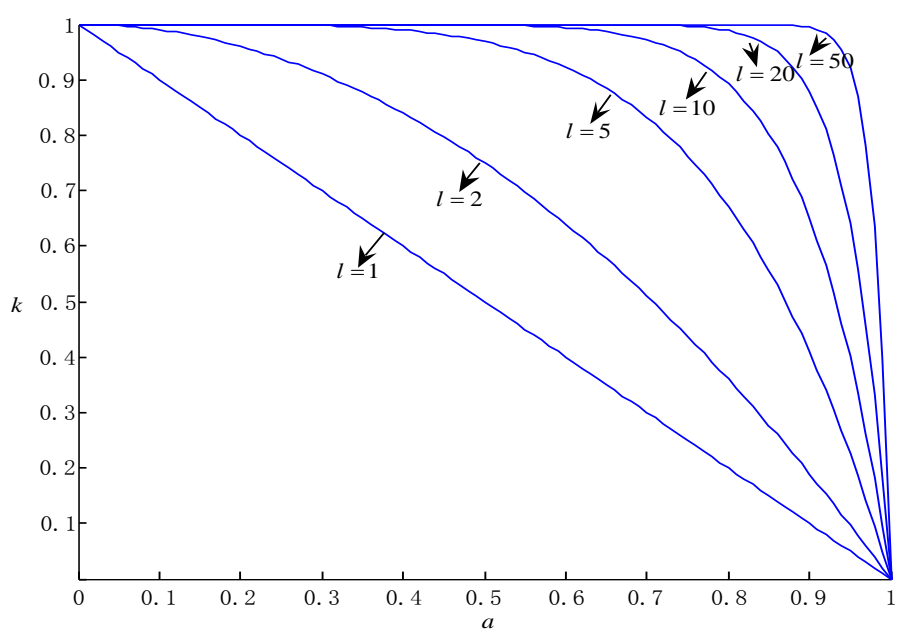

Figure 1. Relationship of $k$ and $a$

Figure 1 showed that when $a$ and $l$ are greater, $k$ is more and more trends to 1 , when $a$ is infinitely close to $0, k$ is infinitely close to 1 , in other words, 1 trust paradox is infinitely close to total conflict paradox.

\section{Ballistic missile target recognition based on expert analysis of trust}

\section{Ballistic missile target recognition systems model construction and analysis}

Ballistic missile target recognition expert system model based on trust shown in Figure 2. As can be seen from the figure, the whole model is divided into four sides which is target layer, evidence processing layer, expert evaluation and decision-making layer.
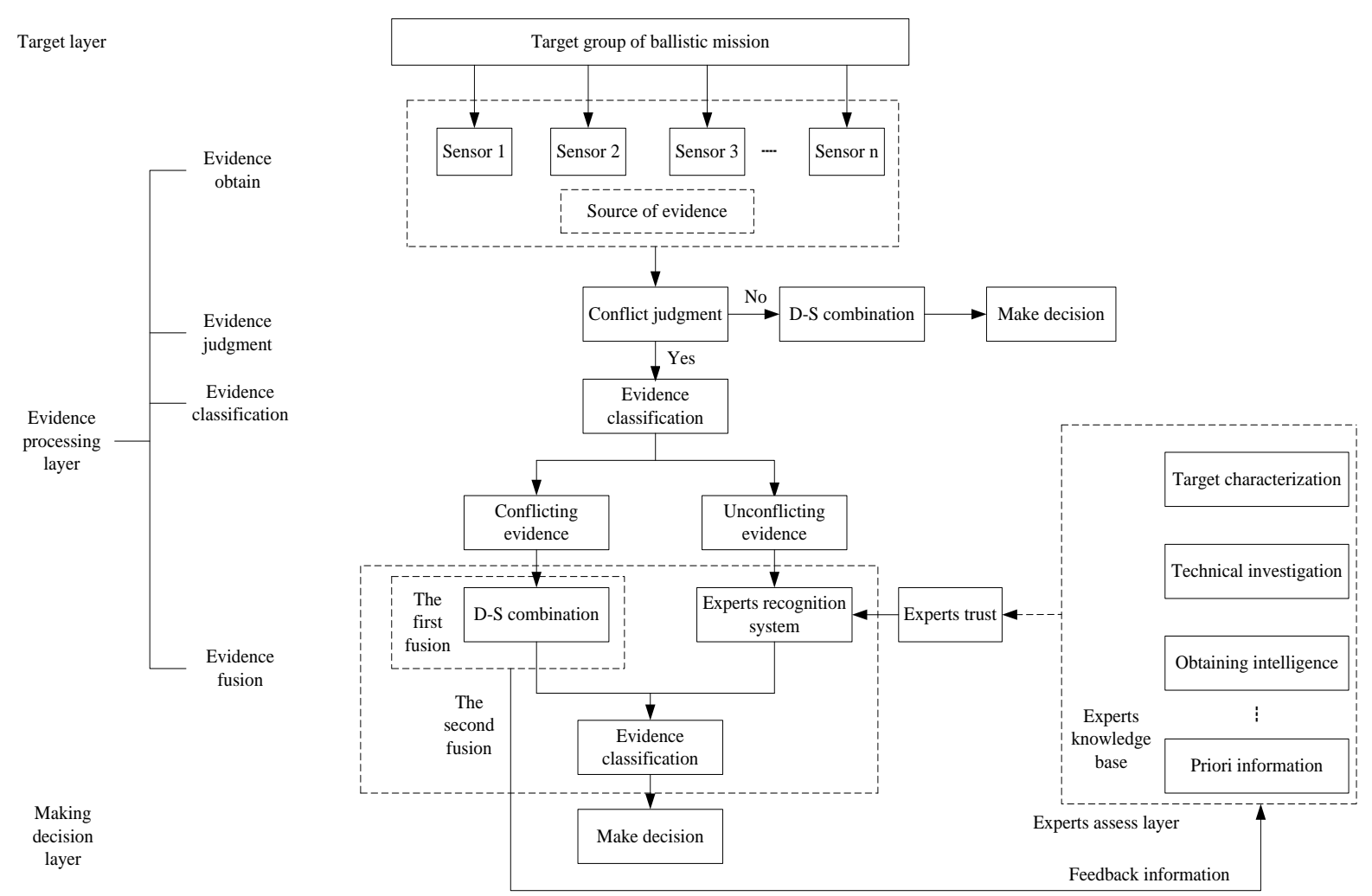

Fig. 2. BM target recognition system based on experts trust 
Target layer clear target object to be identified. Boost phase which goal is relatively simple, but time is short; Middle of the evolution of a single ballistic missile target group and which section lasted longest; Reentry, although lightweight bait, debris , chaff, etc. were filtered with re-entry. Therefore, for the purposes of the entire ballistic missile target recognition system, the difficulty of recognition was how to identify the true ballistic missile warheads from a complex target group.

Evidence processing layer is the core of the model. As can be seen from Figure 2, the evidence processing layer contained evidence obtained, evidence judged and the evidence classification and fusion. The evidence obtained refers to information received from the sensor to obtain the goal of dynamic information; Judgment based on the evidence refers to evidence obtained to determine whether there is a conflict of evidence focused on evidence. Judgment evidence was generally divided into two levels, First, if there is no conflict between the evidence that supports the goal of all the evidence is consistent, then direct the synthesis of D-S, and then make decisions; If there is a conflict between the evidence that there is evidence to support the objective is inconsistent and evidence classification is performed; Evidence classification was foundation of the evidence conflict or not, which collected evidence into conflict and nonconflict of evidence collection set; Evidence based on the integration of sub- categories, generally can be divided into two fusion: The first layer, the integration of non-conflict of evidence between the direct synthesis D-S; The second layer, no conflict of evidence and data synthesized layer is determined by expert assessments conflicting expert evidence trust after its synthesis D-S. It was noted that in the evidence fusion module, and sometimes there will be a multi-layer fusion, that two fusion cannot eliminate the conflict between the evidence, you need to go through multiple layers to give an expert assessment of the experts trust and advance multilayer integration.

Experts assess layer is designed for conflict of evidence set. The core experts assess of layer is an expert knowledge base, the figure 3 showed a model of expert knowledge. As can be seen from Figure 3, expert knowledge composed by a variety of factors, it included target characterization, technical investigation means of obtaining intelligence, information obtained, priori information, sensor location, sensor performance and other factors, each element of the target group at the same time the goal to make a judgment, give target attribute weights, then carry out comprehensive evaluation, at last obtain experts trust target. For an expert knowledge base, the more factors to consider establishing trust experts also more accurate, but at the same time, the more complex of the system, therefore, choosing the appropriate expert knowledge is essential and effective.

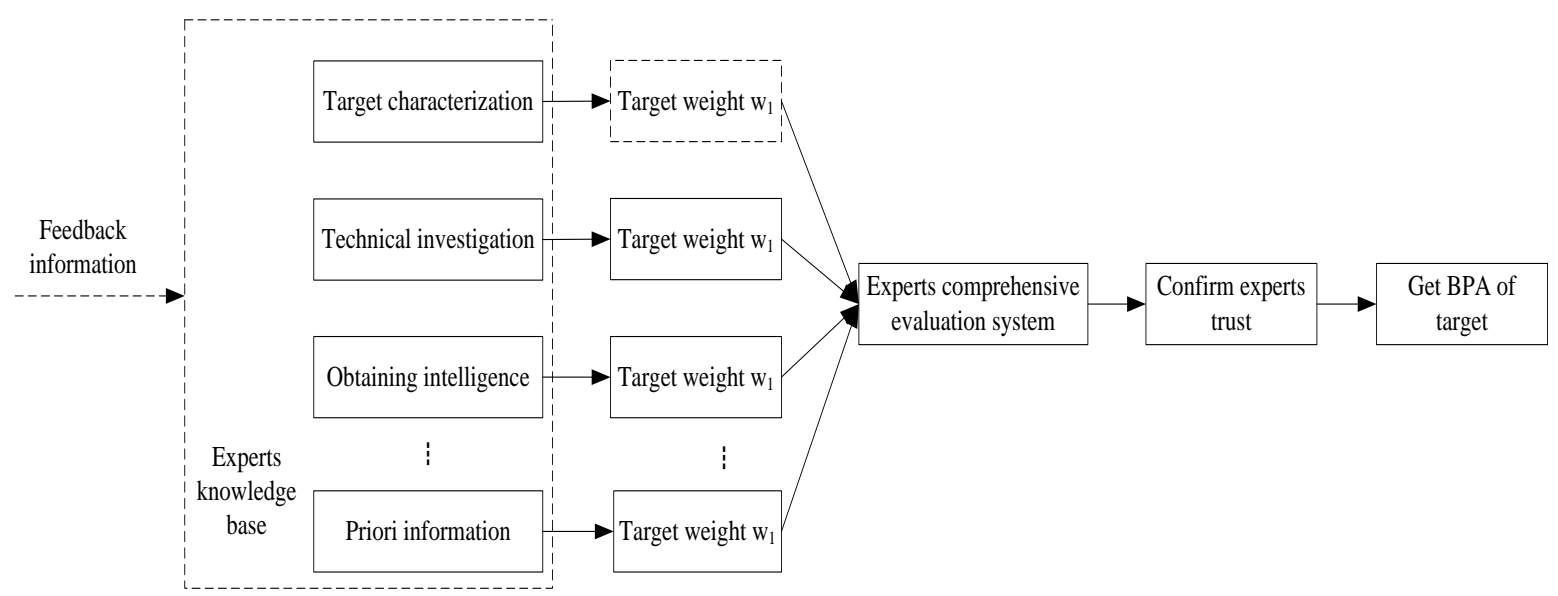

Fig.3. Model of expert knowledge base

By evidence of the results of the decision-making process layer and a layer of synthetic expert assessment, to make an overall judgment on the recognition results, and draw the right conclusions. 


\section{Analysis of recognition method}

From the above analysis we can get the process of target recognition method of ballistic mission based on experts trust:

Step 1: evidence classification. Suppose $\left\{m_{l}\right\}$ is evidence set of $U$ in time $k, m_{\alpha}$ and $m_{\beta}$ belong to $\left\{m_{l}\right\}$ and the same time they are conflicting. Divided $\left\{m_{l}\right\}$ into two categories, one is set of conflicting evidence; the other is set of unconflicting evidence.

Step 2: evidence combination. There two steps in evidence combination, firstly, use D-S combination rule fuse unconflicting evidence set, secondly, use expert knowledge base judge conflicting evidence set and get the BPA of each focal element, which recorded as $m_{\gamma}\left(E_{j}\right)$. There is an expiation that the result of the first step can be seen a feedback information of expert knowledge base.

Step 3: evidence recombination. Use D-S combination rule refuse the results of step 2; get the BPA of each focal element.

Step 4: make decision. According to the results of step 3, determine the target type.

In the air and missile defence system, for a moment the missile target group, a group of sensors in each sensor evidence obtained mostly impossible conflicts, for a mature because of air and missile defence system which immunity should be strong. If evidence of a sensor in each sensor group obtained most of the conflict, then this system not mature enough to effectively complete the task of air and missile defence. Thus, the source of evidence obtained in a certain time, the majority of the evidence is consistent. In other words, once the target recognition, expert evaluation system is very low proportion, which can be understood when evidence of conflict, the subjective judgment of the expert system is an amendment to the objective of the D-S theory, it is also more in line with our objective criterion to judge the facts.

\section{Case Analysis}

According to the requirements of air and missile defense combat system, in this paper, a simulation example used five sensors; it included air surveillance radar $\left(m_{1}\right)$, IFF $\left(m_{2}\right)$, airborne early warning radar $\left(m_{3}\right)$, electronic support measures $\left(m_{4}\right)$, and photoelectric sensors $\left(m_{5}\right)$. The target property of missile target group is true warhead $(A)$, bait $(B)$, other $(C)$, Thus recognition framework we obtained is $U=(A, B, C)$. The typical synthesis rules was Dempster combination rule, Yager combination rule, Sunquan combination rule, Zhang's center combination rule ${ }^{[14]}$, Murphy combination rule, Takahiko's average method combination rule ${ }^{[15]}$. In this paper, compared the text of the proposed method with several typical synthesis rules, and designed 4 kinds of conflict: Lowly conflicting, total conflicting, 0 trust paradox and 1 trust paradox. The simulation results were as follows:

Case1: Lowly conflicting example

Assuming that the target identity BPA obtained at a time by the sensor as shown in table 1 .

TABLE 1 BPA OBTAINED BY SENSORS

\begin{tabular}{|c|c|c|c|c|c|}
\hline Target property & $\boldsymbol{m}_{\boldsymbol{1}}$ & $\boldsymbol{m}_{\mathbf{2}}$ & $\boldsymbol{m}_{\mathbf{3}}$ & $\boldsymbol{m}_{\boldsymbol{4}}$ & $\boldsymbol{m}_{\mathbf{5}}$ \\
\hline$A$ & 0.50 & 0.90 & 0.55 & 0.55 & 0.50 \\
\hline$B$ & 0.20 & 0 & 0.10 & 0.10 & 0.20 \\
\hline$C$ & 0.30 & 0.10 & 0.35 & 0.35 & 0.30 \\
\hline
\end{tabular}

After several synthetic fusion rules, recognition results were shown in table 2.

TABLE 2 RESULT OF LOWLY CONFLICTING

\begin{tabular}{|c|c|c|c|c|c|c|}
\hline \multirow{2}{*}{ Results } & \multicolumn{6}{|c|}{ Methods } \\
\cline { 2 - 7 } & D-S & Yager & SunQuan & Zhang & Murphy & Takahiko \\
\hline$m(A)$ & 0.9841 & 0.0681 & 0.3882 & 0.9841 & 0.9780 & 0.6000 \\
\hline$m(B)$ & 0 & 0 & 0.0640 & 0 & 0.0003 & 0.1200 \\
\hline$m(C)$ & 0.0159 & 0.0159 & 0.1505 & 0.0159 & 0.0217 & 0.2800 \\
\hline
\end{tabular}




\begin{tabular}{|l|l|l|l|l|l|l|}
\hline$m(U)$ & - & - & 0.3973 & - & - & - \\
\hline
\end{tabular}

As can be seen from table II, D-S combination rule and Zhang's center combination rule get better results which reliability rate of real warhead reached 0.9841 , Murphy combination rule also reached 0.978 ; Although average method combination reached 0.6 , from the calculation of view, it showed that with the increasing number of evidence, reliability of unknown set is growing, and not conducive to target recognition. Analysed calculation and rules of rigor of D-S combination rule, Zhang's centre combination rule and Murphy combination rule carefully, it was found that D-S combination rule was the easiest calculation and the most stringent synthesis rule. In low conflict situations, D-S combination rule worked best and highest converge. Therefore, D-S combination rule is the most appropriate in Figure 2.

Case2: Total conflicting example

Assuming that the target identity BPA obtained at a time by the sensor as shown in table 3 .

TABLE 3 BPA OBTAINED BY SENSORS

\begin{tabular}{|c|c|c|c|c|c|}
\hline Target property & $\boldsymbol{m}_{\boldsymbol{1}}$ & $\boldsymbol{m}_{\mathbf{2}}$ & $\boldsymbol{m}_{\mathbf{3}}$ & $\boldsymbol{m}_{\boldsymbol{4}}$ & $\boldsymbol{m}_{\boldsymbol{5}}$ \\
\hline$A$ & 1 & 0 & 0.55 & 0.55 & 0.50 \\
\hline$B$ & 0 & 1 & 0.10 & 0.10 & 0.20 \\
\hline$C$ & 0 & 0 & 0.35 & 0.35 & 0.30 \\
\hline
\end{tabular}

As can be seen from table III, $m_{1}$ and $m_{2}$ completely conflicted. According to the calculation process of Figure 2, divide $m_{3}, \mathrm{~m}_{4}$ and $m_{5}$ into a group, divide $m_{1}$ and $m_{2}$ into a group. After evaluation of the expert system integration, the combination result of $m_{3}, m_{4}$ and $m_{5}$ is $m^{\prime}(A)=0.7961, m^{\prime}(B)=0.0105, m^{\prime}(C)=0.1934$; the combination result of $m_{1}$ and $m_{2}$ is $m_{c}(A)=0.75, m_{c}(B)=0.1, m_{c}(C)=0.15$. Combination results are shown in Table4.

TABLE4 RESULT OF TOTAL CONFLICTING

\begin{tabular}{|c|c|c|c|c|c|c|c|}
\hline \multirow{2}{*}{ Results } & \multicolumn{7}{|c|}{ Methods } \\
\cline { 2 - 8 } & D-S & Yager & SunQuan & Zhang & Murphy & Takahiko & this paper \\
\hline$m(A)$ & - & 0 & 0.2644 & - & 0.9490 & 0.5200 & 0.9521 \\
\hline$m(B)$ & - & 0 & 0.1423 & - & 0.0430 & 0.2800 & 0.0017 \\
\hline$m(C)$ & - & 0 & 0.1017 & - & 0.0080 & 0.2000 & 0.0462 \\
\hline$m(U)$ & - & 1 & 0.4916 & - & - & - & - \\
\hline
\end{tabular}

As can be seen from table 4, D-S combination rule and Zhang's center combination rule could not synthesize the evidence because of the evidence is total conflicting, Yager combination rule could not effectively identify the target which set all conflict assigned to the unknown; Sunquan combination rule would allocate a portion of the conflict to an unknown set, from the process of calculation, when a limited amount of evidence, it could not effectively identify the target. However, Sunquan combination rule could identify the target effectively when valid evidence increases to a certain amount; Murphy combination rule, average combination rule and algorithm in this text could effectively identify target and algorithm of this paper is optimal.

Case3: 0 trust paradox example

Assuming that the target identity BPA obtained at a time by the sensor as shown in table 5

TABLE 5 BPA OBTAINED BY SENSORS

\begin{tabular}{|c|c|c|c|c|c|}
\hline Target property & $\boldsymbol{m}_{\boldsymbol{1}}$ & $\boldsymbol{m}_{\boldsymbol{2}}$ & $\boldsymbol{m}_{\mathbf{3}}$ & $\boldsymbol{m}_{\boldsymbol{4}}$ & $\boldsymbol{m}_{\mathbf{5}}$ \\
\hline$A$ & 0.50 & 0 & 0.55 & 0.55 & 0.50 \\
\hline$B$ & 0.20 & 0.90 & 0.10 & 0.10 & 0.20 \\
\hline$C$ & 0.30 & 0.10 & 0.35 & 0.35 & 0.30 \\
\hline
\end{tabular}

From table 5, we can see $m_{2}$ thought the reliability was 0 but other evidence believes that the target warhead is true. According to the calculation process of Figure 2, divide $m_{1}, m_{3}, m_{4}$ and $m_{5}$ into a group, and $m_{2}$ is another group. From expert systems, combination result of $m_{1}, m_{3}, m_{4}$ and $m_{5}$ was $m^{\prime}(A)=0.8688, m^{\prime}(B)=0.0046, m^{\prime}(C)=0.1266, m_{2}$ was $m_{c}(A)=0.75, m_{c}(B)=0.1, m_{c}(C)=0.15$. Combination results are shown in table 6. 
TABLE 6 RESULT OF 0 TRUST PARADOX

\begin{tabular}{|c|c|c|c|c|c|c|c|}
\hline \multirow{2}{*}{ Results } & \multicolumn{9}{|c|}{ Methods } \\
\cline { 2 - 8 } & D-S & Yager & SunQuan & Zhang & Murphy & Average & this paper \\
\hline$m(A)$ & 0 & 0 & 0.2100 & 0 & 0.7589 & 0.4200 & 0.9710 \\
\hline$m(B)$ & 0.2462 & 0.0004 & 0.1504 & 0.2462 & 0.1411 & 0.3000 & 0.0007 \\
\hline$m(C)$ & 0.7538 & 0.0011 & 0.1411 & 0.7538 & 0.1000 & 0.2800 & 0.0283 \\
\hline$m(U)$ & - & 0.9985 & 0.4985 & - & - & - & - \\
\hline
\end{tabular}

As can be seen from table 6, D-S, Zhang's centre and Sunquan combination rule was assigned to the unknown part of the conflict set while its reliability was 0 for the real warhead, which could not identify the target effectively; Murphy, average method combination rule and algorithm in this text could effectively identify target, and algorithm of this paper is optimal.

Case4: 1 trust paradox example

Assuming that the target identity BPA obtained at a time by the sensor as shown in table 7 .

TABLE7 BPA OBTAINED BY SENSORS

\begin{tabular}{|c|c|c|c|c|c|}
\hline Target property & $\boldsymbol{m}_{\boldsymbol{1}}$ & $\boldsymbol{m}_{\mathbf{2}}$ & $\boldsymbol{m}_{\mathbf{3}}$ & $\boldsymbol{m}_{\boldsymbol{4}}$ & $\boldsymbol{m}_{\boldsymbol{5}}$ \\
\hline$A$ & 0.5 & 0.9 & 0 & 0.9 & 0.5 \\
\hline$B$ & 0.4 & 0 & 0.9 & 0 & 0.4 \\
\hline$C$ & 0.1 & 0.1 & 0.1 & 0.1 & 0.1 \\
\hline
\end{tabular}

It showed that evidence $m_{2}$ and $m_{4}$ thought reliability of the target is bait 0 , sensor $m_{3}$ was thought target identity attribute is true warhead reliability for 0 ; the other two evidence had tended to think that the target warhead true identity attributes. Divide $m_{2}$ and $m_{3}$ into a group, $m_{1}$ and $m_{5}, m_{4}$ into a group. From expert systems, combination result of $m_{1}$ and $m_{5}$ was $m^{\prime}(A)=0.5952, m^{\prime}(B)=0.381, m^{\prime}(C)=0.0238 ; m_{4}$ is $m_{c}(A)=0.6, \quad m_{c}(B)=0.25, \quad m_{c}(C)=0.15$; combination result of $m_{1}, m_{4}$ and $m_{5}$ is $m_{c}^{\prime}(A)=0.7833$, $m_{c}^{\prime}(B)=0.2089, \quad m_{c}^{\prime}(C)=0.0078$; combination result of $m_{2}$ and $m_{3}$ is $m^{\prime \prime}{ }_{c}(A)=0.75, \quad m_{c}{ }_{c}(B)=0.1$, $m^{\prime \prime} c(C)=0.15$. Combination results are shown in table 8 .

TABLE8 RESULT OF 1 TRUST PARADOX

\begin{tabular}{|c|c|c|c|c|c|c|c|}
\hline \multirow{2}{*}{ Results } & \multicolumn{7}{|c|}{ Methods } \\
\cline { 2 - 8 } & D-S & Yager & SunQuan & Zhang & Murphy & Average & this paper \\
\hline$m(A)$ & 0 & 0 & 0.3025 & 0 & 0.9236 & 0.5600 & 0.9638 \\
\hline$m(B)$ & 0 & 0 & 0.1836 & 0 & 0.0762 & 0.3400 & 0.0343 \\
\hline$m(C)$ & 1 & 0 & 0.0540 & 1 & 0.0002 & 0.1000 & 0.0019 \\
\hline$m(U)$ & - & 1 & 0.4599 & - & - & - & - \\
\hline
\end{tabular}

It is noted that example 4 used a multi- expert system evaluation. A trust paradox can be seen as a combination of the whole conflict and trust paradox 0 which looked from the calculation process and Figure 1. If it made $m_{1}, m_{4}$ and $m_{5}$ into a group, and $m_{2}$ and $\mathrm{m} 3$ a group which no effect on the final decision but credit assignment of B would have an impact (At this point $m(B)=0$, this article $m(B)=0.0343$.

It can be seen from table VIII, D-S, Zhang's centre and Sunquan combination rule could not identify the target effectively; Murphy, average method combination rule and algorithm in this text could identify the target effectively, and the algorithm in the text is optimal.

Through the above analysis, Murphy, average method combination rule and algorithm in this text could identify the target effectively in case of conflict of evidence. However, from the algorithm itself, on the one hand, Murphy and averaging combination rule of evidence by averaging, Murphy combination rules need more evidence in order to effectively "offset" collect "bad value" in some cases (As in Example 3, $m_{2}(B)=0.9$, that evidence 2 strongly supported the goal of properties is the bait), but the average method is relatively large fluctuations in combination rule such as example 1, it could be seen from the calculation, the reliability of warheads to average method combination rule was becoming smaller with evidence increasing, 
meanwhile, average method combination rule averaged several independent evidence which is unreasonable in itself; On the other hand, the traditional D-S rule is better than Murphy combination rule and averaging combination rule from the algorithm complexity and rigor of view. In summary, Figure 2 proposed trust based expert system flow chart ballistic missile target recognition is the most reasonable which used traditional synthetic D-S fusion rule, Through the analysis of several common conflict, it also described that the effect of the proposed method was better and had stronger convergence.

\section{Conclusion}

Ballistic missile target recognition was one of the key of anti-missile air defence system construction. The paper aimed the issue of air and missile defence system, multi-sensor system to transmit data conflict, established a ballistic missile target recognition system models and expert knowledge -based trust model expert, proposed ballistic missile target recognition method based on expert trust, and demonstrated the effectiveness of the algorithm through examples, but also existed some problems such as how to determine the experts trust, the robustness of the system model and the timeliness of the system model and so on, which will be the focus of future research.

\section{References}

[1] Ronald R.Yager. On the dempster-shafer framework and new combination rules [J]. Information Sciences, 1987, 41: 93-137.

[2] Dubois D.Prade H. Representation and combination of uncertainty with belief functions and possibility measures [J].Computational Intelligence, 1998, 4(5):244-264.

[3] Sun Quan, Ye Xiuqing, Gu Weikang. A New Combination Rules of Evidence Theory [J]. Acta electronica sinica, 2000, 28(8):117-119.

[4] Lefevre E, Colot O, Vannoorenbergh EP. Belief function combination and conflict management [J].Information Fusion, 2002, 3(3): 149-162.

[5]Deng Yong, Shi Wenkang. A modified combination rule of evidence theory [J].Journal of shanghai jiaotong university,2003, 37(8):1275-1278.

[6] Deng Yong, Shi Wenkang, Zhu Zhenfu. Efficient combination approach of conflict evidence [J].Journal of Infrared and Millimeter Waves, 2004, 23(1): 27-32.

[7] Zhai Haitian, Li Hui, Pan Kai. New evidence composition algorithm based on evidence distance[J]. Modern Electronics Technique,2011,15(34):11-14.

[8] Wang Yongwei, Liu Yunan, Yang Yingjie, et al. New approach for conflict process in D-S evidence theory[J]. Computer engineer and design, 2013,12(34):4316-4320.

[9] Yuan Xiaoke, Wang Xin. Improved synthesis method to D-S evidence theory[J].Computer Measurement \& Control,2013,21(7):1593-1596.

[10] Murphy C. Combining belief functions when evidence conflicts [J]. Decision Support systems, 2000, 29(7):1-9.

[11] Haenni R. Are alternatives to Dempster's rule of combination real alter natives: comments on "about the belief function combination and the conflict management problem" --- Lefevre et al [J]. Information Fusion, 2002, 3(4): 237-239.

[12] Liu Weiru. Analyzing the degree of conflict among belief functions [J].Artificial Intelligence, 2006, 170(11): 909-924.

[13] Jiang Liming, He Jialang, Zhang Hong. New fusion approach for conflicting evidence in D-S theory of evidence [J]. Computer Science, 2011, 38(4):236-238.

[14] Zhang Lianwen. Representation, independence, and combination of evidence in the Dempster-Shafer theory[C]. Yager R R, Kacprzyk J, Fedrizzi M(Eds.). Advances in the Dempster-Shafer Theory of Evidence, Wiley, New York, 1994, 51-69. 
[15] Takahiko H. Decision rule for pattern classification by integrating interval feature values[J]. Pattern Analysis and Machine Intelligence.1998, 20(4):440-447. 